\title{
Resolución de Problemas en Matemática y su Integración con la Enseñanza de Valores Eticos: el caso de Chile
}

\section{Problem Solving in Mathematics and its Integration with the Teaching of Ethical Values: the Chilean case}

\author{
Verónica Díaz Quezada* \\ Alvaro Poblete Letelier**
}

\begin{abstract}
Resumen
Este artículo describe parte de una investigación realizada en la enseñanza secundaria cuyo objetivo fue diseñar una evaluación en el campo del desarrollo valórico considerando la resolución de problemas en matemática, aplicada a 285 estudiantes de segundo año de 23 establecimientos científico-humanista municipales de la Región de Los Ríos y de la Región de Los Lagos de Chile. La metodología usada para su desarrollo fue cualitativa y cuantitativa, e incluyó entrevistas y aplicación de una prueba de situaciones problemas de matemática asociados a valores. Los resultados encontrados evidenciaron la falta de integración de la enseñanza de valores en la formación disciplinaria, además del limitado grado de habilidad de los estudiantes en la resolución de problemas.
\end{abstract}

Palabras-claves: Objetivos Fundamentales Transversales. Valores Éticos. Matemática. Resolución de Problemas.

\begin{abstract}
This article describes part of a research project conducted in secondary education settings whose objective was to design an assessment in the area of values development

\footnotetext{
* Doctora en Educación con Especialización en Matemática por la Universidad Humanismo Cristiano. Académica e investigadora del Departamento de Ciencias Exactas de la Universidad de Los Lagos. Correo postal: Casilla 933, Osorno, Chile.E-mail: mvdiaz@ulagos.cl.

** Doctor en Didáctica Matemática por la Université Laval. Académico e investigador del Departamento de Ciencias Exactas de la Universidad de Los Lagos. Correo postal: Casilla 933, Osorno, Chile. E-mail: apoblete@ulagos.cl.
} 
as regards mathematical problem solving. The assessment was administered to 285 second grade students at 23 city schools with a scientific-humanist focus in Chile's Los Rios and Los Lagos regions. The methodology used for the research was qualitative and quantitative; it included interviews and the administration of a test on math problems associated with values. The results showed a lack of integration of values with the teaching of mathematics, as well as the students' limited problem-solving ability.

Keywords: Cross-Curricular Objectives. Ethical Values. Mathematics. Problem-Solving.

\section{Introducción}

La incorporación de los llamados Objetivos Fundamentales Transversales (OFT) en todas las áreas del currículo en las actuales Reformas Educacionales en Iberoamérica supone un impulso de la formación del alumnado para que sea capaz de construir, racional y autónomamente, su propio sistema de valores, además de enjuiciar de forma crítica la realidad que le rodea e intervenir activamente en su mejoramiento.

La Reforma curricular en Chile ha llamado Objetivos Fundamentales Transversales OFT a aquellos que tienen que ver con la formación general del alumnado y que, por su propia naturaleza, trascienden un sector específico del saber, es decir, son de responsabilidad compartida de muchos espacios curriculares. En ella se explicita que la Educación Secundaria debe orientarse prioritariamente a "la formación del carácter en términos de actitudes y valores fundamentales, misión esencial del Liceo" (MINEDUC, 2011). En la actualidad, la Reforma Educacional vigente en Chile, establece de manera explícita en sus currículum de nivel secundario, que las prácticas educativas en esta etapa incluirán aspectos relativos al desarrollo de las habilidades prácticas, las actitudes y los valores, además de la adquisición de conceptos y conocimientos académicos. Desde esta perspectiva, lo que se intenta fomentar es el desarrollo integral del alumno, dando respuesta a las exigencias de una sociedad moderna que demanda no sólo personas con conocimientos, sino también con sentimientos, autónomos y responsables ante los demás, y con juicio propio ante los acontecimientos cotidianos.

No obstante lo anterior, la evaluación del sistema de valores del alumno aún siendo necesaria no ha sido realizada. Un estudio de evaluación continua del estado del sistema educativo, no sólo debe analizar las adquisiciones académicas sino también los valores adquiridos y las actitudes desarrolladas (DÍAZ; POBLETE, 2006). La medición de estos aspectos conlleva dificultades específicas cuando se trata de grandes muestras. Teniendo en cuenta esta 
limitación, se puede optar por una medida como es la opinión de los estudiantes acerca del grado en que manifiestan conductas tales como agresividad, competitividad etc.

La educación en valores es un tópico que en los últimos tiempos ha sido tema de conversación de familias, comunidades y empresas. No es difícil encontrar quien manifieste que: se han perdido los valores (OROZCO, 2006). Santos (2010) las asocia con las bisagras del sistema y asevera que no están bien engrasadas. Cabe, entonces, preguntarse: $i$ para qué prepara realmente el sistema educativo? ¿para el pensamiento, para el trabajo, para la vida? "El saber siempre es útil, aunque no garantiza necesariamente un trabajo o el éxito. Quizá se haya dado demasiado valor al trabajo y se haya orientado la escuela con referencia a él y a su poder económico" (ANDREOLI, 2008). Se trata de una cuestión esencial. ¿Para qué sirve la escuela? Si pretendemos algo, que conviene precisarlo, es necesario preguntar-se si se está consiguiendo, en qué medida, con qué ritmo y a qué precio. Lo que está claro es que el tema valórico preocupa. La necesidad e importancia del estudio que presentamos radica, por una parte, en que responde a prioridades como tema transversal del sistema de educación, y por otra, ha hecho posible que la resolución de problemas en matemáticas se aplique en función de la formación de valores.

\section{Antecedentes teóricos}

Los nuevos modelos curriculares suelen fundamentarse en la transversalidad, o ejes transversales que se insertan en los currículos con el fin de cumplir objetivos específicos, de proporcionar elementos para la transformación de la educación. Los ejes transversales permiten establecer una articulación entre la educación fundamentada en las disciplinas del saber, los temas y las asignaturas con las carreras de educación superior para formar profesionales integrales. Más concretamente, los valores están presentes en el aula precisamente mediante los temas transversales, estos temas transversales van a responder a realidades o necesidades que tienen una muy especial relevancia para la vida de las personas y la construcción de la sociedad. La formación de valores cobra una importancia vital en estos momentos, pues a escala mundial constituye una preocupación la pérdida de valores que se aprecia, hoy en día, en todas las naciones. Muchos investigadores coinciden en que es imprescindible atender a la formación y fortalecimiento de los valores morales, especialmente en las jóvenes generaciones. 
Investigaciones recientes en campos tan diversos como la filosofía de la educación y la psicología, así como en las ciencias neurobiológicas, han resaltado la necesidad de re evaluar muchas de las suposiciones que respaldan el rol de la escuela, del profesor y de la educación en general. Entre los filósofos destacan los trabajos de Carr $(2003,2006)$ y Kristjánnson (2007) por su constante aplicación de la ética Aristotélica al intentar establecer una base sustentable con una aplicación de integridad filosófica y práctica para la enseñanza. Su trabajo se puede interpretar como una reafirmación del pragmatismo moral de Dewey (1930), quien se basaba en Aristóteles (PAGAN, 2008; PAMENTAL, 2010) al discutir la base moral inherente de la educación. Su renovado interés por Aristóteles surge en medio de un redescubrimiento de la filosofía de la interacción entre la razón y la emoción y la consecuente necesidad para entrenar aquellas emociones (CARR, 2009, 2010, 2011; KRISTJÁNNSON, 2010).

\subsection{Educación en valores y profesión docente}

La educación en valores corresponde a un intento del sistema escolar, por crear nuevas formas de pedagogía y estructuras de apoyo que fomenten el desarrollo de competencias éticas, inclinaciones pro-sociales y competencias en los jóvenes, incluyendo un fortalecimiento de sus objetivos y logros académicos. Recientes estudios han presentado evidencias de las prácticas efectivas que se pueden aplicar ampliamente en el sistema escolar, en distintos niveles y en distintos contenidos. Tras el análisis de investigaciones empíricas se han podido identificar prácticas efectivas, incluyendo prácticas interaccionales, desarrollo profesional, compromiso de los padres, modelos a seguir, y oportunidades de servicio (BERKOWITZ, 2011).

En una revisión de las investigaciones actualizadas encontramos importantes contribuciones a la educación en valores. Tal es el caso de Carr (2011) que explora los aspectos relacionados con los valores en la pedagogía y en la educación docente, distinguiendo tres conceptos o sentidos de valor denominados preferencia de principios, compromiso de principios, y disposición de principios. Para Lovat (2011) la educación en valores es una perspectiva antigua pero a la vez moderna de educación, y tiene un efecto en la calidad de la pedagogía y en el aprendizaje efectivo. Hace referencia a las investigaciones que justifican y explican como la educación en valores funciona para mejorar los efectos positivos en los estudiantes a través del registro completo de medidas de desarrollo personal, emocional, social, moral e intelectual. En la misma línea 
de investigación sobre pedagogía escolar holística y valores, encontramos un estudio realizado en Finlandia, donde el autor ha definido la pedagogía holística en relación con el desarrollo íntegro del estudiante y con la estimulación de las dimensiones sociales, morales, emocionales y espirituales de la educación. El propósito de esta investigación era identificar los componentes de la pedagogía escolar holística y los valores que la sustentan. Después de analizar las entrevistas hechas a los profesores y estudiantes se pudieron identificar tres componentes distintos en la pedagogía escolar holística: valores y las visiones del mundo, componentes pedagógicos que no varían en el campo de estudio y que son importantes independientemente del contenido de la asignatura, y componentes dependientes del campo de estudio, temas o contextos específicos, como el conocimiento de contenido pedagógico de la matemática o el tipo de escuela (TIRRI, 2011).

En Inglaterra exploran la relación binaria entre el desempeño y la moralidad, en relación a una disyuntiva en la forma en que la psicología moral y de desarrollo han influido en la profesión docente. Esto ayuda a tener una mejor comprensión del por qué muchas de las llamadas bases de la enseñanza no han tenido éxito en informar y preparar a los profesores para entregar una educación holística, y a su vez comprender por qué el logro académico de todos los estudiantes sigue siendo un desafío. Arthur y Wilson (2010) exponen un estudio llevado a cabo en el Reino Unido titulado Learning for Life, compuesto por cinco proyectos dirigidos a distintos niveles etarios, y que representa una de las mayores investigaciones sobre educación moral de este tipo que hayan sido desarrolladas en el Reino Unido. El estudio no solo se centra en el carácter sino en las virtudes y valores específicos asociados con este y con su potencial de desarrollo y educabilidad (SOKO; HAMMOND; BERKOWITZ, 2010).

Dasoo (2010), expone un programa Sudafricano diseñado para inculcar valores pedagógicos en los profesores y para tratar el gran impacto demostrado por el aumento en la autoestima y bienestar de los profesores a medida que experimentaron una mejora en las respuestas de aprendizaje de los estudiantes como resultado de las prácticas pedagógicas. Crotty (2010), recurre a una perspectiva Habermasiana para darle sentido al foco académico mejorado, que tan claramente se vio demostrado en los estudios de caso presentados en el Australian Journal of Teacher Education en relación a los estudiantes involucrados en la pedagogía valórica. Esta perspectiva le permitió nominar el efecto como un pensamiento superior que conllevó a un conocimiento emancipatorio; una forma de aprendizaje superior, que Habermas $(1972,1974)$ 
llamó conocimiento humano auténtico. De esta perspectiva, Crotty (2010) concluye que no sorprende que el desarrollo de tales capacidades de conocimiento pudiera tener efectos de flujo en el desempeño académico.

Algunos estudios que brindan una propuesta conceptual reforzada y una verificación empírica de las interconexiones inherentes entre la pedagogía valórica y el bienestar holístico del estudiante, incluyendo una mejora académica, muestran un aumento en el número y en alcance (NUCCI; NARVAEZ, 2008; ARTHUR; WILSON, 2010; LOVAT; TOOMEY; CLEMENT, 2010; LOVAT et al., 2011). Entre estas investigaciones, una evidencia fundamental es la obtenida en las investigaciones y en la aplicación de los proyectos procedentes del Programa de Educación Moral de Australia, en el que se concluyó que, basado en la evidencia, la pedagogía valórica tiene el potencial de afectar positivamente el ambiente de aprendizaje total de una escuela, lo que origina ciertas características, incluyendo un fortalecimiento en la relación profesor-alumno, clima, actitudes y valores de la sala de clases, actitudes y comportamientos de los estudiantes, conocimiento y entendimiento de los estudiantes, así como sus logros. El Informe del Estado 2 (DEEWR, 2010) identificó conexiones más claras y sofisticadas entre la presentación de la pedagogía valórica y los efectos en el comportamiento y en el desempeño de los estudiantes.

La evidencia de Australia y de otros países muestra claramente que el objetivo del tipo de educación holística identificado en las Declaraciones de Adelaide y Melbourne ha sido de utilidad para la pedagogía valórica. La evidencia también sugiere que tal psicología tiene un efecto significativo en los sistemas escolares en varias partes del mundo (NUCCI; NARVAEZ, 2008; ARTHUR, 2010; DEEWR, 2010; LOVAT; TOOMEY; CLEMENT, 2010).

Hoy en día, las escuelas luchan por preparar a sus estudiantes para los desafíos, de carácter moral y de desempeño, que les depara el siglo XXI. Es de igual importancia reforzar el desempeño académico, reducir las tasas de deserción, o mejorar la integridad, seguridad, desarrollar un carácter y una cultura de excelencia y valórica puesto que son de gran importancia en el sistema escolar. $\mathrm{Y}$ sin embargo, esto se debe hacer con limitaciones de dinero y tiempo, en un mar de prioridades y requerimientos y sin el apoyo del currículo, capacitación o profesionales que se necesitan.

El estado soviético tampoco se ha sustraído a la preocupación de educar en valores, es así como el sistema educacional de Valily Sukhomlinsky (19181970) se toma como ejemplo de una comunidad ética de aprendizaje. Uno de las mayores preocupaciones de Sukhomlinsky fue la de inculcar en sus estudiantes 
los valores de compasión y servicio, combinado con la sensibilidad hacia la belleza en la naturaleza, al arte y hacia las relaciones humanas. Para Sukhomlinsky, existieron miles de conexiones entre la educación física, moral, estética, intelectual y vocacional. Su enfoque hacia la educación en valores es inusual, en el sentido que se aplicó en todo el programa escolar y que se combinó con la capacitación práctica en relación al buen desempeño (COCKERILL, 2011).

Entre las investigaciones internacionales también están las de Benninga et al. (2006) quienes usaron el Índice Académico de California como guía para mostrar una correlación entre el desarrollo de valores de alta calidad (carácter) y el fortalecimiento de los logros académicos. Davidson, Lickona e Khmelkov (2007); Davidson, Khmelkov e Lickona (2010) explican y demuestran una correlación similar en su conexión del carácter de desempeño y del carácter moral, relacionados integralmente con el desarrollo de la personalidad. Osterman (2010) presenta más evidencias de estos efectos combinados al mostrar que es el profesor quien enseña contenidos de calidad en el contexto de la pedagogía efectiva y establece relaciones positivas para desarrollar ricas relaciones valorativas en los estudiantes para a través de estos valores mejorar sus efectos académicos.

A la luz de la abundante evidencia disponible en relación a los efectos de la pedagogía valórica en el desarrollo de los estudiantes a través de las distintas medidas, es quizás sorprendente que la educación docente no muestre más señales de ajustarse para acomodar esta evidencia. Esto ha ocurrido en ocasiones (TOOMEY et al., 2010), pero en su mayor parte, la educación docente se presenta como una industria conservadora, conocida más por su reactividad y pro-actividad; igualmente, es poco probable que haya una respuesta generalizada de la educación docente hasta que los sistemas educativos se saturen en la pedagogía valórica y/o en las prácticas pedagógicas similarmente orientadas, junto con sus supuestos de aprendizaje y resultados.

\subsection{La resolución de problemas y los valores}

En matemática, como sector de aprendizaje, la presencia de los OFT, exigen que desde la práctica docente se contribuya a la formación para la vida, conjugando en un todo integrado el desarrollo intelectual con la formación éticosocial de los alumnos. En los programas de la enseñanza secundaria en matemática, los OFT del ámbito del desarrollo del pensamiento, postulan de manera explícita, la necesidad de desarrollar habilidades de investigación y de 
resolución de problemas, ya que

[...] a través de los problemas rutinarios a resolver matemáticamente, que plantean las actividades del programa de matemática, es posible ampliar el trabajo de los Objetivos Transversales Fundamentales con estudiantes a su capacidad de juicio, y la aplicación de criterios morales, a problemas del medio ambiente, económicos y sociales (MINEDUC, 2000, p. 13).

En el marco de la Asociación Internacional para la Evaluación del Rendimiento Educacional IEA se lleva a cabo el International Mathematics and Science Study TIMSS. Se explicita en el TIMSS 2003 la exigencia de resolver problemas enmarcados en contextos matemáticos o de la vida real de los que es muy poco probable que los alumnos hayan encontrado ítemes similares (BEATON et al., 2003). En tanto que, en el marco de la Organización para la Cooperación y el Desarrollo Económico OCDE se lleva a cabo el Programme for International Student Assessment PISA, el cual constituye un estudio sobre el conocimiento, las destrezas, y las competencias de carácter amplio, importantes para la vida, que se encuentran incorporadas de manera transversal en el contexto de las asignaturas de lenguaje, ciencias y matemática. El PISA, al igual que el TIMMS, enfatiza la valoración de las competencias transversales, entre las que destaca la capacidad para solucionar problemas.

En la actualidad, en Iberoamérica, tanto en los artículos de didáctica de la matemática como en los textos de matemáticas, se reconoce a la resolución de problemas como un modelo de actividad por excelencia. En Chile, la resolución de problemas también constituye un elemento fundamental en la enseñanza actual de la matemática en los diversos niveles, y gran parte de su justificación la reciben de su necesidad de aplicación y utilidad en la vida cotidiana (DÍAZ; POBLETE, 2009). Como habilidad fundamental y en relación con los contextos, constituye uno de los objetivos importantes del sistema escolar, habilitando a los alumnos a enfrentarse con tareas no previstas y a encontrar algún tipo de respuesta adecuada. Proyectos experimentales en didáctica de las matemáticas han demostrado que dar contextos significativos no es solamente un estímulo para aprender, sino también constituye un medio para crear estrategias (ROGOFF et al. (1997) en BAZZINI, 2001).

Concretamente, el entorno social en que viven los alumnos ofrece un contexto apropiado para hacer matemáticas. También existen variadas clasificaciones de problemas al igual que muchos modelos de resolución de 
problemas que van desde los clásicos propuestos por Polya (1982) y Schoenfeld (1988), próximos a la heurística y otros menos matemáticos y más psicológicos de Bransford y Stein (CHAMORRO, 2003). La conveniencia de encontrar una determinada heurística que ayude a la resolución de los problemas, llevó a explicitar un cierto número de estrategias generales de resolución, susceptibles de facilitar el trabajo y a reconocerla como un recurso metodológico que puede contribuir poderosamente a desarrollar las capacidades de los alumnos (GARCÍA, 2002).

Es evidente que la resolución de problemas continúa siendo un tema importante del currículum y más aún en la perspectiva de los temas transversales de las reformas educativas emprendidas en los últimos años. Esta consideración respecto a la actividad de resolución, ha sido objeto de reconocimiento en diversas reformas educacionales en Iberoamérica, pero ¿es posible afirmar que los profesores están habituados a realizar una enseñanza de la disciplina, donde se manifieste además de lo conceptual y procedimental, lo valórico? A partir de esta pregunta de investigación y centrados en los OFT, formulamos los siguientes objetivos : diseñar una evaluación en el campo del desarrollo valórico, considerando la resolución de problemas en matemática, desarrollar un estudio cualitativo de los aspectos valóricos, en segundo año de Educación Secundaria e integrar la enseñanza de valores y la resolución de problemas en matemáticas.

Las hipótesis de trabajo que se formularon fueron las siguientes:

H1: Existe una falta de desarrollo de competencias en resolución de problemas asociadas a situaciones valóricas.

H2: Existe una falta de incorporación de valores a través de la enseñanza de la matemática

En este artículo, por razones de espacio y cantidad de información, se presenta sucintamente el desarrollo cualitativo y resultados cuantitativos obtenidos en este proyecto.

\section{Metodología}

Este trabajo forma parte de un proyecto de investigación de mayor envergadura (Fondecyt $N^{\circ} 1040035$ ) financiado por la Comisión Nacional de Investigación Científica y Tecnológica de Chile CONICYT, y se llevó a cabo en el área de Didáctica de la Matemática de la Universidad de Los Lagos. Corresponde a un estudio descriptivo exploratorio con desarrollo de metodología cualitativa y cuantitativa. 
Se consideró como población a los estudiantes de la enseñanza media de 23 establecimientos estatales con modalidad científico- humanistas municipalizados, pertenecientes a las regiones de Los Ríos y de Los Lagos de Chile que comprenden siete provincias. La muestra fue escogida al azar por muestreo aleatorio simple y la constituyeron 285 alumnos de 15 años de edad, que cursaban el segundo año de la enseñanza secundaria en Chile. A este nivel, durante el segundo semestre del año lectivo 2011, se les aplicó instrumentos evaluativos los que previamente fueron validados en su totalidad por contenido mediante el juicio de expertos en el tema de educación y de matemática, aceptando un grado de concordancia entre ellos mayor o igual a $85 \%$.

Con la finalidad de obtener a través de la opinión de los estudiantes, una medida directa de la apropiación de valores y su relación con la resolución de problemas de matemática propuestos, se realizaron tres entrevistas la totalidad de los alumnos, distribuidos de manera proporcional entre las siete provincias sujetas a estudio. Las entrevistas contenían 78 preguntas referidas a situaciones valóricas y 8 preguntas relacionadas con el conocimiento de los ejes transversales, las cuales se transcribieron literalmente en notas de cada sesión de investigación. Estas entrevistas incluían, por una parte, la presentación de situaciones relativas a los 16 valores siguientes: educación sexual, educación para la salud, educación ambiental, educación para la paz, autonomía, autoestima, solidaridad y compañerismo, tolerancia y respeto a los demás, sinceridad, responsabilidad, honradez, libertad personal, autenticidad y coherencia, bondad, y por otra, preguntas relativas a los ejes transversales.

También se les aplicó una prueba construida con doce situaciones problemas de matemática asociados a valores. Las situaciones problemas fueron previamente piloteadas y aceptadas para la prueba definitiva sólo aquellas en que se logró un porcentaje mayor o igual a 70\% de resultados positivos, con lo cual se conformó la versión final con 12 problemas idóneos al nivel, ni muy fáciles ni muy difíciles, sino representativos de las habilidades características en matemáticas de los alumnos típicos del ciclo medio. Se diseñó esta prueba de modo que se pudiesen conocer además de la competencia de los alumnos en la resolución de problemas, el grado en que los profesores de matemática integran la enseñanza de valores en los contenidos de la disciplina. Esta contenía situaciones problemas de matemática que involucraban valores específicos tales como solidaridad, compañerismo, educación para la salud, educación ambiental, tolerancia y respeto a los demás, justicia, trabajo en equipo, responsabilidad, respeto a la naturaleza, bondad y cuidado personal, los cuales debían ser 
reconocidos por ellos a través de los problemas presentados.

Los niveles de logro se consideraron en relación al grado de avance de los estudiantes en la resolución de los problemas matemáticos, y se estimó de acuerdo al modelo de Rasch (NCTM 1980, adaptado por Díaz y Poblete, 1998) en una misma escala con la dificultad de los problemas. Este modelo postula una interacción entre una persona y un problema, colocando el desempeño personal en función de la habilidad de la persona y la dificultad del problema presentado.

Se establecieron los grados de discriminación interna a través de la correlación biserial y los de dificultad para cada problema y para la prueba en su totalidad, previa definición de los criterios estadísticos aceptados universalmente para tal ejecución. La confiabilidad de la prueba basada en la homogeneidad, se estimó con el coeficiente Alfa de Cronbach y fue 0,89 considerados altamente adecuados dada la naturaleza del instrumento evaluativo y su extensión.

\section{Resultados}

Tanto las entrevistas como la aplicación de la prueba de situaciones problemas de matemática, asociados directamente a valores, aportaron al conocimiento del grado de desarrollo de la habilidad de resolución de problemas en matemática, y al conocimiento de la importancia que los establecimientos educacionales a través de su proyecto educativo le dan a la profundización de valores considerados fundamentales en la Reforma Educativa vigente. El análisis que se lleva a cabo a continuación, considera solo algunos de los problemas diseñados y propuestos en forma personal a los estudiantes.

\subsection{Prueba de situaciones problemas de Matemática}

A continuación se presentan ejemplos de situaciones problemas y sus correspondientes respuestas por parte de los estudiantes.

\section{Situación Problema}

En una clínica, tres médicos atienden semanalmente sin costo a un total de 75 pacientes de cáncer. Si el primer médico atendiera 5 pacientes más, trabajaría tanto como el segundo médico, pero si el tercer médico cediera 5 pacientes, atenderían la misma cantidad de personas que el segundo.¿Cuántas personas atiende en forma gratuita cada uno?

¿Con qué valores asocias la situación problema planteada?

Silvia: con el valor de la solidaridad; Gricell: igualdad, porque a veces las 
personas no cuentan con dinero suficiente para pagar el costo de un médico; Iris: con el respeto y el amor hacia las personas porque las está ayudando; Oscar: la pregunta indica que los médicos se esmeran para hacer su trabajo

Situación Problema

Los nutricionistas preocupados por la obesidad de nuestro país, elaboraron un gráfico que muestra la cantidad mínima de calorías por hora y por $\mathrm{m}^{2}$ de superficie corporal, que se necesita en función de la edad (o años corresponde a un recién nacido). Hace dos siglos, el matemático Lagrange desarrolló un polinomio interpolar que permite "fabricar" una función polinómica que pase por los puntos que se desee. Por ejemplo, con los 5 puntos del gráfico se puede construir la siguiente función: $\mathrm{C}(\mathrm{x})=-0,0046 \times 4+0,2 \times 3-2,95 \times 2+16,36 \mathrm{x}+30$ donde $\mathrm{x}$ es la edad en años y c las calorías necesarias. ¿Cuántas calorías necesita un adolescente de 14 años? ¿Qué ocurre con los requerimientos calóricos durante los primeros 5 años de vida?

¿Con qué valores asocias la situación problema planteada?

Silvia: "Con el valor de la salud" Iris: "Con la amabilidad, porque hay una preocupación hacia los niños obesos". Jacqueline: "Se asocia más que nada con la preocupación de una persona con otra". Oscar: "Mejorar la alimentación”.

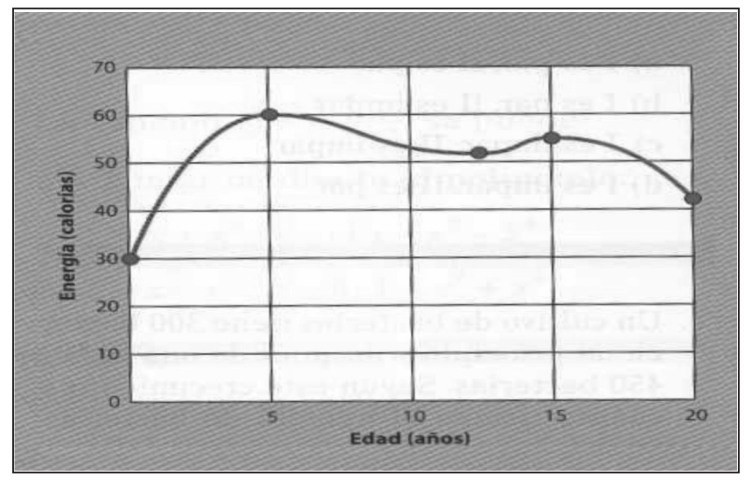

Figura 1 - Calorías en función de la edad

Ante la consulta realizada en cada una de las situaciones problemas descritas anteriormente: sin importar el contenido matemático, ¿trabajas regularmente en matemática con problemas que incluyen situaciones valóricas como esta?, responden lo siguiente: Yoselin: no, porque siempre la profesora está haciendo problemas con números y letras y nada más; Jacqueline: no 
siempre, sólo de vez en cuando; Edwin: no, generalmente en este año no trabajamos con tantos problemas, pero en años anteriores si, pero estas no contenían situaciones de valores.

Analizadas todas las respuestas de la prueba asociadas a valores, podemos indicar que los estudiantes sólo lograron relacionar adecuadamente en un $35,7 \%$ los valores que se asocian a cada uno de las situaciones problemas de matemática. El porcentaje restante, es decir el $64,3 \%$, se distribuye entre aquellos estudiantes que no reconocen adecuadamente el valor involucrado y los que declaran no saber con que valor asociar la situación matemática planteada. Sin importar el contenido matemático, el 74,2\% de los estudiantes, reconoce no trabajar regularmente en matemática con problemas que incluyen situaciones valóricas.

\subsection{Niveles de logro}

Los grados de logro obtenidos por los estudiantes en la prueba de situaciones problemas, se presentan a continuación en la Figura 2.

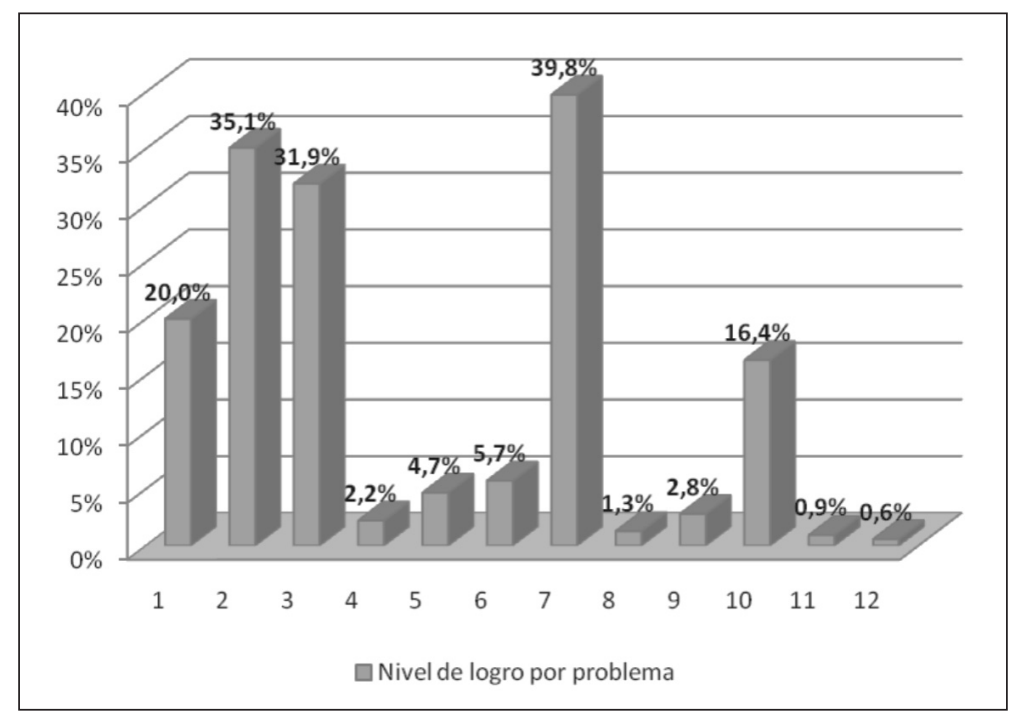

Figura 2 - Nivel de logro por problema

El nivel de logro en promedio alcanzado por la totalidad de los estudiantes fue de $13,45 \%$. Resultados que dada la naturaleza del instrumento evaluativo se consideran inadecuados, colocando en evidencia que los estudiantes no tienen 
claridad de cómo utilizar los conceptos matemáticos en una situación concreta. Se agrega, además, la falta de conocimientos en cuanto a propiedades y utilización de fórmulas lo que les dificulta la resolución de los problemas, y deja de manifiesto que la posibilidad de hacer matemáticas en el aula ocupando la resolución de problemas con una cierta cercanía a contextos familiares para los estudiantes, no es tarea fácil.

\subsection{Entrevistas}

Con la finalidad de obtener una medida directa a través de la opinión de los estudiantes acerca del grado en que reconocen conductas relativas a valores, se realizaron tres entrevistas individuales a los estudiantes. A continuación se presenta en la Figura 3 el porcentaje de apropiación de los valores obtenidos por los estudiantes a partir del análisis de las entrevistas.

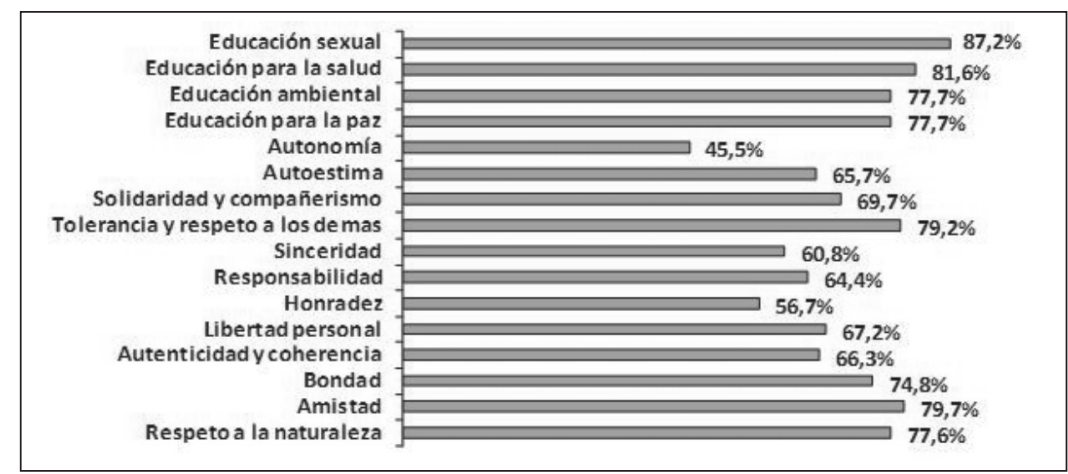

Figura 3 - Apropiación de los valores en los estudiantes

De acuerdo a los resultados de las entrevistas, como se muestra en la Figura 3, podemos indicar que las tres mayores apropiaciones de los valores descritos, corresponden a educación sexual, educación para la salud y amistad. Las menores apropiaciones de valores corresponden a autonomía, honradez y sinceridad.

Considerando la totalidad de las situaciones planteadas en las entrevistas referidas a la educación para la salud, los estudiantes lograron en promedio un 81,6\% de manifestación de apropiación de este valor. Ante la consulta: ¿te preocupas por tener una alimentación equilibrada? son ejemplos de sus respuestas:

Jonathan: no me preocupa para nada; Marcelo: siempre, es más por 
tener una buena salud y sobre todo por verse bien y no preocupar a mis padres sobre todas las cosas.

Cuando se pregunta: ¿te molesta que no existan políticas públicas que permitan prevenir o disminuir los factores de riesgo provocados por el consumo de tabaco y la obesidad?, indican: Luis: si, eso es algo que le falta mucho a nuestro país, ya que no tenemos claro los riesgos de prevención; Sergio: si, ya que si existiera algo para evitar el consumo del tabaco y la obesidad, se podrían evitar varias cosas, como por ejemplo enfermedades, etc.

En lo referente a la educación sexual, el porcentaje medio de apropiación de este valor en los estudiantes es de 87,2 \%. Ante las consultas: ¿te interesas por asistir a charlas sobre sexualidad?”, “ ¿consideras importante que se enseñe educación sexual en tu liceo?; ¿estás de acuerdo en que el uso de preservativos es necesario? y ¿tienes conocimiento del SIDA y sus efectos?, la totalidad considera importante que se les enseñe educación sexual en su Liceo, al igual que están completamente de acuerdo en el uso del preservativo. Respecto al conocimiento que tienen del SIDA y sus efectos, dan como respuesta: Natali : aquí en el Liceo nos han hablado mucho de lo que es educación sexual, iniciación sexual y todo, o sea como se dice vulgarmente a calzón quitado y nos han dicho lo que es SIDA, nos han dicho sus causas, sus consecuencias y puchas tenemos más que claro eso, incluso en la clase de biología nos dividen por grupos y nos hacen tocar temas así.

En lo referente a la Amistad, del total de respuestas obtenidas en las entrevistas a los estudiantes, el porcentaje medio de apropiación de este valor logrado por ellos es $79,7 \%$. Son ejemplos de las preguntas las siguientes: ¿consideras que los amigos son necesarios?; ¿ estás de acuerdo en que a tu edad tener amigos no es importante? y ¿prefieres tener muchos más conocidos que pocos amigos? A las cuales responden: Mario: sí, sirven de mucho sobre todo cuando uno está mal o más lo necesita; Edgard: si, porque uno se siente muy bien cuando tiene un amigo, te puede ayudar, aconsejar, etc.

Cuando se les consulta: ¿crees tu que es obligación de los amigos ayudarte cuando tu estás en problemas?, dan respuestas como: Alejandro: si es realmente mi amigo, va a tener que ayudarme por su propia voluntad.

En los planteamientos que dan cuenta de la apropiación del valor autonomía, los estudiantes lograron sólo un 45,5 \% de manifestación de apropiación del valor. En preguntas referidas a la búsqueda de textos por su propia cuenta 
para apoyarse en sus estudios, a la toma de decisiones y su dependencia de otros, a la posibilidad de investigar por sí mismos, dan respuestas como las siguientes: Jonathan: no, porque no tengo mucho tiempo para ir a la biblioteca, pero cuando quiero conseguirles a mis friends, se me olvida; Luis: prefiero que me enseñen porque ahí puedo aprender, uno sólo muchas veces no puede.

En el valor honradez, se obtiene un porcentaje promedio de $56,7 \%$. Cuando se les consulta: ¿crees tu que si tienes la oportunidad de copiar en una prueba es natural aprovecharla?, dan respuestas tales como: Daniela obvio, si no estudié y si se da la oportunidad se aprovecha; Héctor: si porque los profesores las están dando; Francisco: la mayoría copia en las pruebas, pienso que no debe ser porque si uno quiere ser alguien en la vida depende de si mismo y no de un amigo u otra persona.

Frente a la pregunta ¿estás de acuerdo en que no es tan malo quedarse con lo ajeno cuando tu lo necesitas?, responden: Julio: estoy completamente de acuerdo; Valeria: si lo necesito, no es tan malo, pero igual queda el cargo de conciencia.

Ante la siguiente situación planteada a los estudiantes: ¿supongamos que estás a cargo de una compra para tu curso y el vendedor se equivocó a tu favor, ¿consideras necesario aclarar el error?, dan respuestas como: Miriam: obvio que aclararía el error; Andrea: no, porque así puedo gastar el vuelto demás sin que nadie se dé cuenta; Víctor: depende si gano mucho $o$ pierdo poco.

En lo referente a la Sinceridad, de las respuestas obtenidas en las entrevistas a los estudiantes, el porcentaje medio de apropiación logrado por ellos en este valor es $60,8 \%$. Cuando se les consulta si están de acuerdo en que decir siempre la verdad es un problema, dan respuestas como las siguientes: Claudia: decir siempre la verdad hace que la persona sea sincera y se le genera un gran respeto hacia ella; Jaime: no es que sea un problema, es que a veces decir la verdad puede resultar perjudicable por lo que una mentira puede salvarte.

En la tercera entrevista se les consultó a los estudiantes sobre su conocimiento de los OFT, a lo cual respondieron mayoritariamente que no los conocían. Del mismo modo, de manera mayoritaria, consideran que los valores y las actitudes positivas son esenciales para su desarrollo personal, tales como la responsabilidad, sinceridad, honradez, amistad, humildad, respeto a las personas, solidaridad, bondad, lealtad, autoestima y generosidad. 
Para los estudiantes, los valores se desarrollan a su juicio en prácticamente todas las actividades del Liceo, destacando las conversaciones y la religión como las instancias que contribuyen a su desarrollo. De acuerdo a sus respuestas relativas al fomento de los valores en los consejos de curso, las opiniones están divididas. En lo referente a la oportunidad que tiene el profesor de matemáticas para fomentar los valores y actitudes positivas, además de dar tareas en matemática que los involucran, los estudiantes responden mayoritariamente: $s i$, pero cuando hay malos comportamientos; el profe de matemática como que nos aconseja más sobre nuestro futuro o sea nos deja claro que si tu no estudias vas a ser un fracasado en la vida, También responden: no, no me han dado tareas así; son ejercicios, pero no involucran valores.

Una parte minoritaria de los estudiantes manifiestan que durante la clase de matemáticas tienen oportunidad de demostrar sus valores y actitudes positivas, y dan como argumento lo siguiente: si, ayudando o explicándole a mis compañeros cuando no saben realizar los ejercicios; si, conversando más con el profesor y tratando otros tipos de temas, mientras que otros, no tienen una respuesta. Finalmente, responden mayoritariamente que el profesor no se apoya constantemente en contenidos matemáticos para fomentar valores y actitudes positivas.

\section{Conclusiones y discusión}

Del análisis de las respuestas de los estudiantes participantes de nuestro estudio, podemos inferir que las clases de matemáticas no están dando a los estudiantes las herramientas para entender mejor su realidad, por ende el programa de matemáticas debiera ser mejorado mediante el establecimiento de un entorno en el que los alumnos estén expuestos a la enseñanza a través de la resolución de problemas.

Este estudio deja en evidencia un importante déficit en el desarrollo de la habilidad para resolver problemas en matemática y en el tratamiento de los objetivos o ejes transversales, ya que como enseñanzas dotadas de objetivos y contenidos específicos, que deben ser asumidas por el currículum en su conjunto, no están del todo integradas en la estructura curricular en matemáticas, con lo cual no contribuyen significativamente a fortalecer y afianzar la formación valórica de los estudiantes, ni a orientar el proceso de crecimiento y autonomía personal y la forma en que los estudiantes se relacionan con otras personas y con la sociedad. Este estudio refleja que los valores no se tienen en cuenta en las 
programaciones de aula, tampoco se trabajan en unidades didácticas específicas en matemática, sólo son trabajados en momentos concretos, con lo cual se transforman en actividades puntuales y episódicas no insertas de manera medular en el currículo del establecimiento educacional.

Específicamente en matemáticas y de acuerdo a los resultados evidenciados, podemos afirmar que los profesores no están habituados a realizar una enseñanza de la disciplina, donde se manifieste además de lo conceptual, lo valórico. Por otra parte, el planteamiento de situaciones problemas y la muy poca capacidad para resolverlos, dejó en evidencia la falta de desarrollo de la habilidad de resolución de problemas, además de la falta de incorporación y apropiación de valores a través de la asignatura. Por tanto se comprobaron ambas hipótesis de estudio.

Aún cuando el estudiante se manifiesta dándole importancia a determinados valores, no es capaz de reconocerlos en las situaciones problemas de matemática planteadas. Además, aseguran no trabajar regularmente en matemática con problemas que incluyen situaciones valóricas, lo cual deja de manifiesto una perdida de oportunidad del profesor, para formular situaciones problemas de matemática que impliquen la apropiación efectiva de valores, que en el caso de la autonomía, honradez y sinceridad, resultan ser los valores que menor apropiación de ellos, manifiestan los estudiantes de enseñanza secundaria.

A la luz de este estudio, de manera general podemos afirmar, por una parte, que presentar un problema y desarrollar las habilidades necesarias para resolverlo es más motivador que la enseñanza de las habilidades sin un contexto, ya que permite a los estudiantes encontrar una razón para aprender matemáticas y por tanto participar más activamente en el aprendizaje de la misma, y por otra, resulta imprescindible que la adquisición de conocimientos académicos, la formación social y la formación en valores, conformen un todo del proceso educativo, y de manera particular, que la Educación Secundaria puede y debe, al igual que la familia, hacer una contribución importante en el plano valórico promoviendo en los estudiantes la capacidad para ejercer con responsabilidad grados de libertad y autonomía, realizar actos de generosidad y solidaridad, en un marco de reconocimiento y respeto, donde las convicciones personales puedan convivir con el respeto a las actitudes y valores de los demás. 


\section{Referencias}

ANDREOLI, V. Carta a un profesor. Barcelona: Integral, 2008.

ARTHUR, J. Of good character: Exploring virtues and values in 3-25 year olds. Exeter, UK: Academic Imprint, 2010.

ARTHUR, J.; WILSON, K. New research directions in character and values education in the UK. In: LOVAT, T.; TOOMEY, R.; CLEMENT, N. (Eds.). International research handbook on values education and student wellbeing. Dordrecht, Netherlands: Springer, 2010. p. 339-358.

BAZZINI, L. Las dos caras de las matemáticas en Italia: propuestas para la educación básica. In: GIMENEZ, J. (Org.). Matemáticas en Europa: diversas perspectivas. Barcelona: Grao, 2001. p. 34-46.

BEATON, A.; MULLIS, I. V. S.; MARTIN, M. O.; GONZÁLEZ, E. J.; KELLY, D. L.; SMITH, T. A TIMSS Assessment Frameworks and Specifications 2003. TIMSS International Study Center 2003. Chestnut Hill, MA: Boston College, 2003.

BENNINGA, J.; BERKOWITZ, M.; KUEHN, P.; SMITH, K. Character and academics: What good schools do. Phi Delta Kappan, Bloomington, v. 87, p. 448-452, Jan. 2006.

BERKOWITZ, M. What works in values education. International Journal of Educational Research, Durham, v. 50, n. 3, p. 153-158, 2011.

CARR, D. Character and moral choice in the cultivation of virtue. Philosophy, Cambridge, v. 78, n. 2, p. 219-232, Mar. 2003.

CARR, D. Professional and personal values and virtues in education and teaching. Oxford Review of Education, London, v. 32, n 2., p. 171-183, Aug. 2006.

CARR, D. Virtues, mixed emotions and moral ambivalence. Philosophy, Cambridge, v. 84, n. 1, p. 31-46, Jan. 2009.

CARR, D. Personal and professional values in teaching. In: LOVAT, T.; TOOMEY, R.; CLEMENT, N. (Eds.). International research handbook on values education and student wellbeing. Dordrecht: Springer, 2010. p. 63-74.

CARR, D. Values Education and Holistic Learning. International Journal of Educational Research, Durham, v. 50, n.3, p. 171-176, 2011.

COCKERILL, A. Values education in the Soviet State: The lasting contribution of V.A. Sukhomlinsky. International Journal of Educational Research, Durham, v. 50, n. 3, p. 198-204, 2011. 
CROTTY, R. Values education as an ethical dilemma about sociability. In: LOVAT, T.; TOOMEY, R.; CLEMENT, N. (Eds.). International research handbook on values education and student wellbeing. Dordrecht: Springer, 2010. p. 631-644.

CHAMORRO, M. Las dificultades de lectura y comprensión de los problemas matemáticos escolares. UNO: Revista de Didáctica de las Matemáticas, Barcelona, v. 33, p. 99-119, June 2003.

DASOO, N. Nurturing teacher wellbeing through values education. In: LOVAT, T.; TOOMEY, R.; CLEMENT, N. (Eds.). International research handbook on values education and student wellbeing. Dordrecht: Springer, 2010. p. 359-376.

DAVIDSON, M.; LICKONA, T.; KHMELKOV, V. Smart and good schools: A paradigm shift for character education. Education Week, Bethesda, v. 27, n. 12, p. 32-40, Nov. 2007.

DAVIDSON, M.; KHMELKOV, V.; LICKONA, T. The power of character: Needed for, and developed from, teaching and learning. In: LOVAT, T.; TOOMEY, R.; CLEMENT, N. (Eds.). International research handbook on values education and student wellbeing. Dordrecht: Springer, 2010. p. 427-454.

DEEWR. Giving voice to the impacts of values education: The final report of the Values in Action Schools Project. Canberra: Australian Government Department of Education, Employment and Workplace Relations, 2010. Disponible en: http://www.valueseducation.edu.au/values/val_vasp_final_report,30121.html. Acceso en: 24. Mar. 2013.

DEWEY, J. The quest for certainty: A study of the relation of knowledge and action. London: George Allen \& Unwin, 1930.

DÍAZ, V.; POBLETE, A. Resolver tipos de problemas matemáticos: ¿una habilidad inhabilitante?. Revista Epsilon, Sevilla, v. 14, n. 42, p. 409-423, dic. 1998

DÍAZ, V.; POBLETE, A. Objetivos transversales en situaciones problemas en matemáticas: una evaluación en reforma. Revista Investigaciones en Educación, Temuco, v. vi, n. 1,p. 129-146, jul. 2006.

DÍAZ, V.; POBLETE, A. Competencias y transposición didáctica: binomio para un efectivo perfeccionamiento en matemática. Revista Investigación y Postgrado, Carácas, v. 24, n. 2, p. 77-107, ago. 2009.

GARCÍA, E. Resolución de problemas y desarrollo de capacidades. UNO: Revista de didáctica de las Matemáticas, Barcelona, v. 29, p. 20-38, mar. 2002. 
HABERMAS, J. Knowledge and human interests. Tradução de J. Shapiro. London: Heinemann, 1972.

HABERMAS, J. Theory and practice. Tradução de J. Viertal. London: Heinemann, 1974.

KRISTJÁNNSON, K. Aristotle, emotions and education. Aldershot: Ashgate, 2007.

KRISTJÁNNSON, K. The self and its emotions. Cambridge: Cambridge University Press, 2010.

LOVAT, T. Values education and holistic learning: Updated research perspectives. International Journal of Educational Research, Durham, v. 50, n. 3, p. 148-152, 2011. Disponible en:

<http://www.sciencedirect.com/science/journal/08830355>. Acceso en: 24 mar. 2013.

LOVAT, T.; TOOMEY, R. (Eds.). Values education and quality teaching: The double helix effect. Dordrecht, Netherlands: Springer, 2009.

LOVAT, T.; TOOMEY, R.; CLEMENT, N. (Eds.). International research handbook on values education and student wellbeing. Dordrecht: Springer, 2010.

LOVAT, T.; DALLY, K.; CLEMENT, N.; TOOMEY, R. Values pedagogy and student achievement: Contemporary research evidence. Dordrecht, Netherlands: Springer, 2011.

MINEDUC. Ministerio de Educación Chile. Educación Secundaria. Formación General. Santiago: Impresos Universitaria, dic. 2011.

MINEDUC. Ministerio de Educación Chile. Objetivos Fundamentales y Contenidos Mínimos Obligatorios de la Educación Secundaria. Santiago: Impresos Universitaria, oct. 2000.

N.C.T.M. Problem Solving in School Mathematics. Virginia: Preston, 1980.

NUCCI, L.; NARVAEZ, D. (Eds.). Handbook of moral and character education. New York: Routledge, 2008.

OROZCO, J. Valores, Juventud, Sociedad y Futuro. Revista Educación en Valores, Valencia, v. 2, n 6, p. 55-61, dic. 2006.

OSTERMAN, K. F. Teacher practice and students' sense of belonging. In: LOVAT, T.; TOOMEY, R.; CLEMENT, N. (Eds.). International research handbook on values education and student wellbeing. Dordrecht: Springer, 2010. p. 239-260. 
PAGAN, N. Configuring the moral self: Aristotle and Dewey. Foundations of Science, Netherlands, v. 13, n. 3, p. 239-250, Sep. 2008

PAMENTAL, N. Dewey, situationism, and moral education. Educational Theory, Illinois, v. 60, n. 2, p. 147-166, Apr. 2010.

POLYA, G. How To Solve it. New Jersey: Princeton University Press, 1982.

SANTOS, M. Una pretensión problemática: educar para los valores y preparar para la vida. Revista de Educación, Madrid, v. 35, n. 351s, p. 36-47, abr. 2010.

SCHOENFELD, A. Problem Solving in Context(s). In: CHARLES, R.; SILVER, E. (Ed). The Teaching and Assesing of Mathematical Problem Solving. Reston, VA: The National Council of Teachers of Mathematics, 1988. p. 32-60.

SOKO, B.; HAMMOND, S.; BERKOWITZ, M. The developmental contours of character. In: LOVAT, T.; TOOMEY, R.; CLEMENT, N. (Eds.). International research handbook on values education and student wellbeing. Dordrecht: Springer, 2010. p. 579-604.

TIRRI, K. Holistic school pedagogy and values: Finnish teachers' and students' perspectives. International Journal of Educational Research, Durham, v. 50, n. 3, p. 159-165, 2011.

TOOMEY, R.; LOVAT, T.; CLEMENT, N.; DALLY, K. (Eds.). Teacher education and values pedagogy: A student wellbeing approach. Sydney: David Barlow Publishing, 2010.

Submetido em Maio de 2012. Aprovado em Setembro de 2012. 


\section{ANEXO 1}

\section{Cuestionario de Situaciones Problemas de Matemática}

Los Objetivos Fundamentales Transversales que se proponen en la Educación Secundaria, hacen referencia, como finalidad general, a las actitudes y valores que se espera que tu como estudiante desarrolles en el plano ético.

En relación a esta formación valórica, te solicitamos RESOLVER LOS PROBLEMAS y reconocer los VALORES, tales como libertad, honradez, sinceridad, etc. que se asocian a cada una de las situaciones-problemas de matemática presentadas.

Instrucción: Resuelve el problema planteado y responde a las preguntas en la línea punteada

\section{Situación Problema 1}

A dos amigos se les ha ocurrido una idea para iniciar una cadena y llevar alimento a un hogar de ancianos. Cada uno de ellos, ha pedido 1 kilo de legumbre, a dos de sus amigos. Un día después, cada uno se lo ha pedido a otros dos amigos. Estos, a su vez, lo volvieron a pedir a otros dos. Y así sucesivamente, durante 10 días. ¿Cuántos kilos de legumbres deberían recaudar?. Si hubiesen llamado a 3 amigos, ¿cuántos kilos habrían recaudado en 5 días?

1) ¿Con qué valores asocias la situación problema planteada?

2) Sin importar el contenido matemático, ¿trabajas regularmente en matemática con problemas que incluyen situaciones valóricas como esta?

3) Resuelve el problema planteado

\section{Situación Problema 2}

Para broncearse en el verano, se recomienda partir con 15 minutos de exposición al sol el primer día e ir gradualmente aumentando un minuto cada día, hasta un máximo de media hora. Si $\mathrm{t}=14+\mathrm{d}$, donde $\mathrm{t}$ representa el tiempo y d el número de días, ¿cuánto tiempo puedes broncearte al décimo día?. ¿en cuántos días completas la exposición al sol? Si lo que deseas es un bronceado total y para ello debes exceder las recomendaciones aumentando peligrosamente el tiempo de exposición al sol, ¿te arriesgarías?

\section{Situación Problema 3}

Para pavimentar las calles con hormigón armado, los albañiles diseñan previamente una serie de rectángulos congruentes entre sí.

En muchas ciudades de Chile, las calles de las poblaciones más pobres no están pavimentadas. Esto trae como consecuencia una mayor contaminación, producto del polvo en suspensión, y grandes dificultades en el invierno, puesto que las calles se transforman en un barrial por donde es muy difícil transitar.

Para solucionar estos problemas existe un programa de pavimentación comunitario, en el cual los vecinos aportan el 10\% del costo si se trata de una calle, y al menos un $20 \%$ si se trata de un pasaje y el resto lo provee el Ministerio de Vivienda y Urbanismo. 
El costo aproximado de 100 metros de calle es de $\$ 12.000 .000$, si en ella viven 24 familias que deben aportar el 10\% del total, ¿cuánto aporta cada familia.

Si los rectángulos tienen 5 metros de largo y con 2 de ellos se cubre el ancho de la calle: ¿Cuántos rectángulos son necesarios para pavimentar los 100 metros?

\section{Situación Problema 4}

La pirámide de Keops tiene una base cuadrada de $233 \mathrm{~m}$ por lado y una altura de $146 \mathrm{~m}$. Se estima que trabajaron en ella alrededor de 100.000 hombres durante 20 años. Si suponemos que solo descansaban 10 horas, entre dormir y comer, y trabajaban todos los días de la semana:

¿cuánto demorarían los mismos 100.000 hombres en las condiciones actuales, es decir, tomando 260 días hábiles y 8 horas diarias de trabajo?

\section{Situación Problema 5}

A raíz de una inundación, se desea ayudar a pintar casas de emergencia. Para ello se deben considerar algunos factores como los metros cuadrados que se van a pintar, litros de pintura necesarios y costo de la pintura.

Tomando en cuenta que el modelo de una casa es como el de un cubo de $5 \mathrm{~cm}$. de arista, y que cada galón de pintura alcanza para x metros cuadrados y tiene un valor de p pesos cada uno, encuentra el costo de este trabajo.

\section{Situación Problema 6}

Si la superficie total afectada por incendios forestales de los bosques en la Regiones de Los Lagos y de Los Ríos por temporada entre los años 2006 a 2010 es el siguiente:

\begin{tabular}{|l|c|c|c|c|c|}
\hline Región & 2006 & 2007 & 2008 & 2009 & 2010 \\
\hline Total País & 40.081 & 43.595 & 90.888 & 101.690 & 17.179 \\
\hline $\begin{array}{l}\text { Regiones de Los Lagos } \\
\text { y de Los Ríos }\end{array}$ & 16.000 & 901 & 40.722 & 3.703 & 149 \\
\hline
\end{tabular}

Cuál es el porcentaje de incendios forestales en la región de Los Lagos con respecto al país? Represéntalo en un gráfico circular. Determina el valor promedio de incendios en todo el país.

\section{Situación Problema 7}

Don Luis está organizando una cena para la navidad a la que asistirán 8 personas, por lo que necesita comprar 3 kilos de carne. Se entera que la familia vecina no tendrá cena para esta ocasión, y decide invitar a los 4 integrantes que componen esta familia. ¿Cuántos kilos más de carne deberá comprar Don Luis?

\section{Situación Problema 8}

Los nutricionistas preocupados por la obesidad de nuestro país, elaboraron un gráfico que muestra la cantidad mínima de calorías por hora y por $\mathrm{m}^{2}$ de superficie corporal, que 
se necesita en función de la edad (o años corresponde a un recién nacido)

Hace dos siglos, el matemático Lagrange desarrolló un polinomio interpolar que permite "fabricar" una función polinómica que pase por los puntos que se desee. Por ejemplo, con los 5 puntos del gráfico se puede construir la siguiente función:

$C(x)=-0,0046 x^{4}+0,2 x 3-2,95 x^{2}+16,36 x+30$ donde $x$ es la edad en años y $\mathrm{C}$ las calorías necesarias.

¿Cuántas calorías necesita un adolescente de 14 años?

¿Qué ocurre con los requerimientos calóricos durante los primeros 5 años de vida?

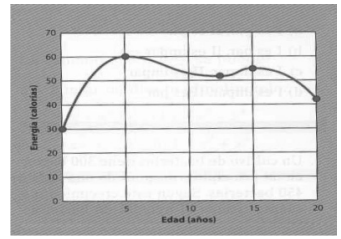

\section{Situación Problema 9}

En Chile, las estadísticas indican que por cada cuatro chilenos que no fuman, hay tres que si lo hacen. Si se sabe que la población actual es cercana a los 15.750 .000 habitantes, determinar el valor aproximado de cuántas personas son fumadores. Determinar cuántas personas son las llamadas "fumadores pasivos" que aunque no fumen directamente, aspiran el humo que desechan los fumadores, siendo aún más perjudicial ya que contiene los residuos más tóxicos del cigarrillo.

\section{Situación Problema 10}

En una clínica, tres médicos atienden semanalmente sin costo a un total de 75 pacientes de cáncer. Si el primer médico atendiera 5 pacientes más, trabajaría tanto como el segundo médico, pero si el tercer médico cediera 5 pacientes, atenderían la misma cantidad de personas que el segundo. ¿Cuántas personas atiende en forma gratuita cada uno?

\section{Situación Problema 11}

Un bebe en un año usa en promedio 800 pañales desechables, lo que equivale a cortar 4 árboles para su fabricación. Al estudiar una muestra durante un año de 125.000 bebes, se concluyó que: un $30 \%$ no usa pañales desechables, un $20 \%$ usa 400 pañales desechables y el resto usa solamente pañales desechables. ¿Cuántos árboles se cortaron en un año?

\section{Situación Problema 12}

En un determinado período, María realiza en total 130 acciones de higiene como las siguientes: se lava los dientes, se ducha y lava su ropa. Sabiendo que el número de veces que se lava los dientes, representa los $5 / 2$ del número de veces que se ducha, y el número de veces que lava su ropa, equivale a los 2/45 del lavado de dientes. ¿Cuántas veces durante el período María realiza cada actividad de higiene? 